\title{
Combining material and model pedigree is foundational to making ICME a reality
}

\author{
Steven M Arnold*, Frederic A Holland Jr, Brett A Bednarcyk and Evan J Pineda
}

\author{
* Correspondence: \\ Steven.M.Arnold@nasa.gov \\ NASA Glenn Research Center, 21000 \\ Brookpark Road, Cleveland, $\mathrm{OH}$ \\ 44135, USA
}

\begin{abstract}
With the increased emphasis on reducing the cost and time to market of new materials, the need for analytical tools that enable the virtual design and optimization of materials throughout their processing-internal structure-property-performance envelope, along with the capturing and storing of the associated material and model information across its life cycle, has become critical. This need is also fueled by the demands for higher efficiency in material testing; consistency, quality, and traceability of data; product design; engineering analysis; as well as control of access to proprietary or sensitive information. Fortunately, materials information management systems and physics-based multiscale modeling methods have kept pace with the growing user demands. Herein, recent efforts to identify best practices associated with these user demands and key principles for the development of a robust materials information management system will be discussed. The goals are to enable the connections at various length scales to be made between experimental data and corresponding multiscale modeling toolsets and, ultimately, to enable ICME to become a reality. In particular, the NASA Glenn Research Center efforts towards establishing such a database (for combining material and model pedigree) associated with both monolithic and composite materials as well as a multiscale, micromechanics-based analysis toolset for such materials will be discussed.
\end{abstract}

Keywords: Information Management; Informatics; Data schema; Analysis; Experimental Data; Simulation Data; Pedigree; Multiscale Modeling; Micromechanics

\section{Background}

With the increased emphasis on reducing the cost and time to market of new materials, ICME (Integrated Computational Materials Engineering) has become a fast-growing discipline within materials science and engineering. The vision of ICME is compelling in many respects, not only for the value added in reducing time to market for new products with advanced, tailored materials but also for enhanced efficiency and performance of these materials. Although the challenges and barriers (both technical and cultural) are formidable, substantial cost, schedule, and technical benefits can result from broad development, implementation, and validation of ICME principles [1]. ICME is an integrated approach to the design of products, and the materials that comprise them, by linking material models at multiple time and length scales.

A key ingredient is the linkage with manufacturing processes, which produce internal material structures, and in turn influence material properties and allowables, enabling tailoring (engineering) of materials to specific industrial applications. Figure 1 illustrates 


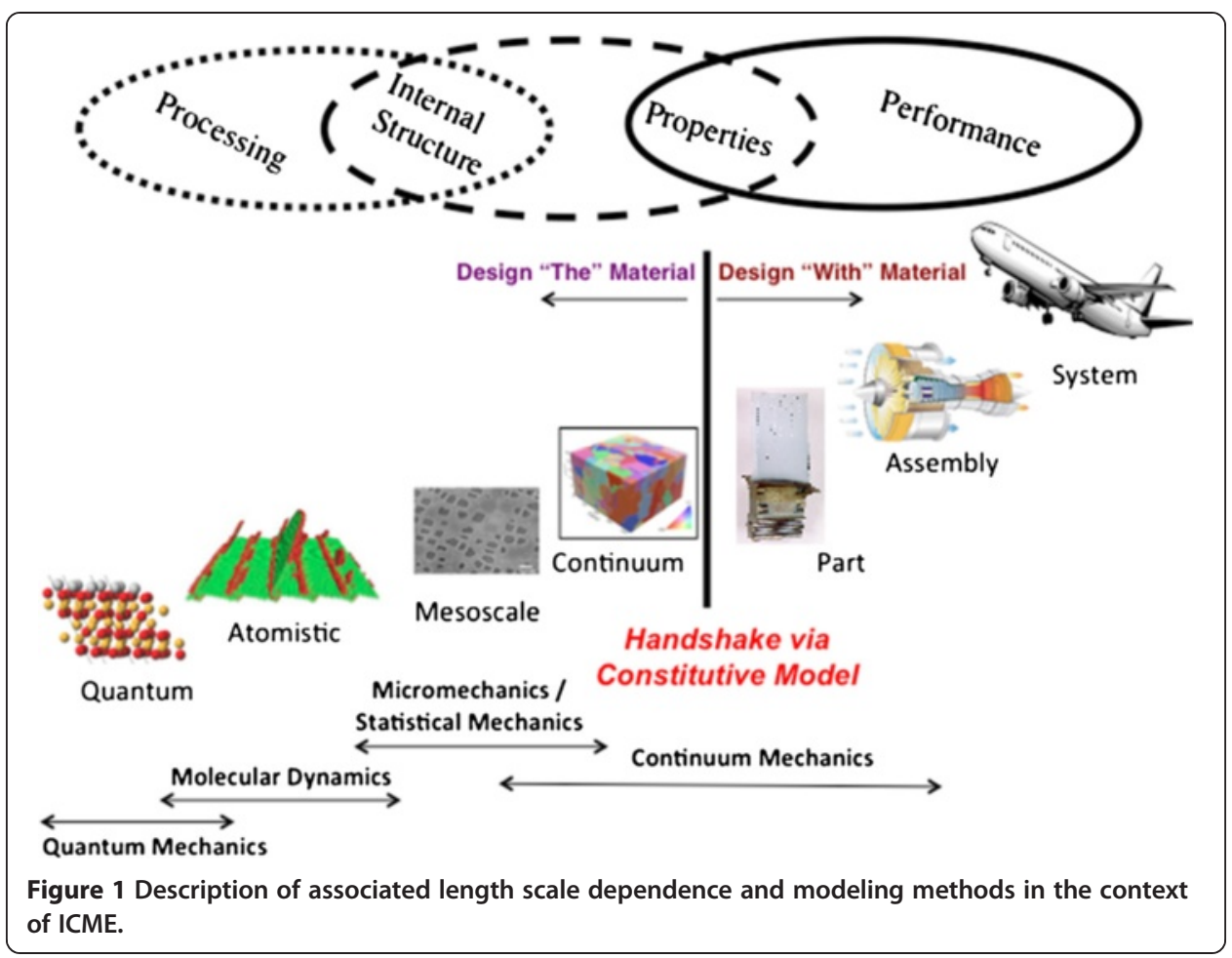

the interconnection of these scales and their cause/effect relationships, e.g., processing conditions produce a particular microstructure from which properties are obtained, which then dictate a specific structural performance. Note that the evolution of elliptical line types (i.e., dotted to dashed to solid line) are purposely included to imply the level of maturity/understanding (from immature, to semi-mature, to mature, respectively) of modeling at each level of scale (both temporal and geometric). Furthermore, the figure illustrates the difference between two non-exclusive viewpoints, that is, designing 'withthe-material' (structural analyst viewpoint) versus designing 'the material' (a materials scientist viewpoint). It is also apparent that the fundamental linkage between these two viewpoints is ultimately the associated constitutive model(s) for a particular material. One cannot overestimate the importance of understanding the input and output at each scale in order to determine the appropriate 'handshaking' between scales and the meaningful properties that are ultimately required by a structural analyst.

Equally important is the fact that experiments (whether computational/virtual or laboratory) performed at a given level can be viewed from two perspectives. If one 'looks up' to higher scales, then the results can be viewed as exploration or characterization experiments used to identify/obtain the necessary model features or parameters, respectively, operating at the present and/or next higher level. Conversely, if one 'looks down', these same results can be used to validate the modeling methods/approaches employed to transition from the lower level(s) to the given level.

While there is a clear indication that ICME is growing, utilization of ICME in the daily work of researchers and engineers is still lacking. The key contributing factors, since ICME is an inherently data-intensive activity, are the lack of a robust information management system and the lack of a digital storage culture within most organizations. This stems from the fact that on the surface, a materials properties database may seem 
simply like a fancy means of storing, retrieving, and distributing materials data, something akin to an electronic file cabinet. However, as discussed by Marsden et al. [2] and Arnold et al. [3], an effective ICME materials database (e.g., one in which experimental and computational mechanics are fully coupled) must allow the data inside a database to be seamlessly accessible by analysis tools and allow the results from analyses to be read back into the database and stored with all of the associated metadata, while keeping track of associations across the full range of length scales.

For example, for a physics-based model to predict the yield strength of a nickel-based superalloy it may need to draw upon quantum mechanics predictions of stacking fault energies, lattice distortions, and phase equilibria of several different alloying elements. These predictions would be combined with microstructural scale models that either use the quantum mechanics predictions or are calibrated with experimental data. Phase equilibria models such as CALPHAD ${ }^{\circ}$ models are an example, as well as processingmicrostructure models of castings or forgings. Important information necessary for a yield strength model would include not only equilibrium phases but also the kinetics of microstructural evolution (of several features, including $\gamma^{\prime}$ precipitate and carbide size and spacing, grain size and grain boundary phases). The maturity of these models already allows semi-quantitative predictions of various parameters, but the development of higher fidelity models will require the capture, analysis, and dissemination of higher fidelity data, as well as all associated pedigree information for calibration and validation. For example, while a current model may utilize an average particle size as a key parameter, future models may require the entire particle size and shape distributions to be measured and tracked with respect to various manufacturing methods. Clearly, the enormity of data types (e.g., discrete, functional, structured, and unstructured) and the sheer quantity of data can be overwhelming. Consequently, historical static data systems are likely to be gradually phased out, evolving to become an integral part of dynamic materials property databases that are web-accessible and in which data - and the relationships between items of data - can be interactively searched, reorganized, analyzed, and applied. These dynamic databases have great superiorities in satisfying the needs of modern materials-related sciences and engineering focused activities like ICME.

Furthermore, it is critical to understand that ICME is not just developing processingmicrostructure (P-M) relationships or microstructure-property (M-P) relationships independently, rather it is the full integration of these various length scale-specific relationships, wherein linkages from processing all the way up to performance can be made and utilized. This requirement greatly increases the need for data/metadata and contextual linkage so that knowledge can be both captured and discovered. For example, the variety and complexity of modern materials, and their applications, necessitate complicated, and often extensive, materials testing. As for composite materials, large volumes of test data on various forms of the composites themselves, as well as individual constituents' thermal and mechanical behavior, are often required. Given a micromechanics-based analysis approach, it is typical to require that data for each constituent be reliably and conveniently traced back from the final products through their processing steps to the original raw materials. A second example is the need to provide adequate data to support increasingly sophisticated nonlinear, anisotropic, and multi-scale engineering analyses. Here again, instead of storing a simple set of reduced, point-wise data, like elastic modulus and yield strength, the entire response (e.g., stress-strain, creep, 
and relaxation) curve may be required. Collating, storing, processing, interacting with, and finally applying such data and metadata require advanced dynamic information systems, enabling management of changing proprietary data alongside reference data collections, while ensuring consistency, quality, applicability, and traceability.

Prior publications [3-6] discussed the data scheme, best practices, and informatics required to establish a robust, twenty-first century information management system for capturing and analyzing materials information. The goal of the information management system is to enable 1) generalized constitutive modeling and 2) data mining to establish microstructure/property/failure relationships for monolithic and composite materials. The proposed schema/requirements for ICME were demonstrated using a turbine disk Ni-based superalloy, in Arnold et al. [3]. Furthermore, Arnold et al. [6] argued that integrating both virtual (computationally based) and experimental data, over the entire material data life cycle (see Figure 2) and at various length scales, in the same information management system is essential for ICME to become a reality and to permeate the material and engineering cultures within a given organization. For example, Figure 3 illustrates the interaction between experimental data and virtual data (data resulting from simulation tools) in that some experimental processing data $(A)$ serves as input to a process model which in turn outputs some microstructural feature $(W)$, which is stored in the database. This virtual microstructure data is then combined with measured microstructural data $(B)$ and provided as input to a micromechanics and/or statistical mechanics analysis package, which then generates materials property data $(X, Y)$, which again is stored in the database. This property data $(X, Y)$ is then subject to experimental validation $(E, F)$ and also used in some continuum-level analysis package (e.g., finite element analysis (FEA)) to assess some

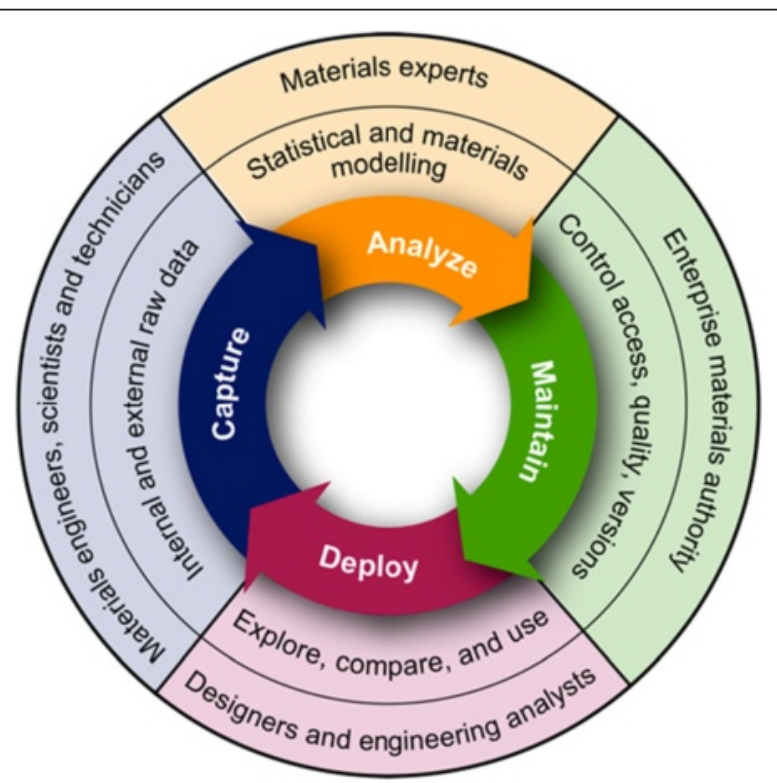

Figure 2 Four aspects of material data life cycle as defined by the MDMC. The Material Data Management Consortium (MDMC) is a group of aerospace and energy sector organizations (both industrial and governmental) that have joined forces to develop best practices and associated software tools to integrate material and structural information technology with the realities of practical product design and advanced research. This group was established in 2002 through collaboration with ASM International, NASA Glenn Research Center, and Granta Design Limited [16] (see www.mdmc.net [17]). 


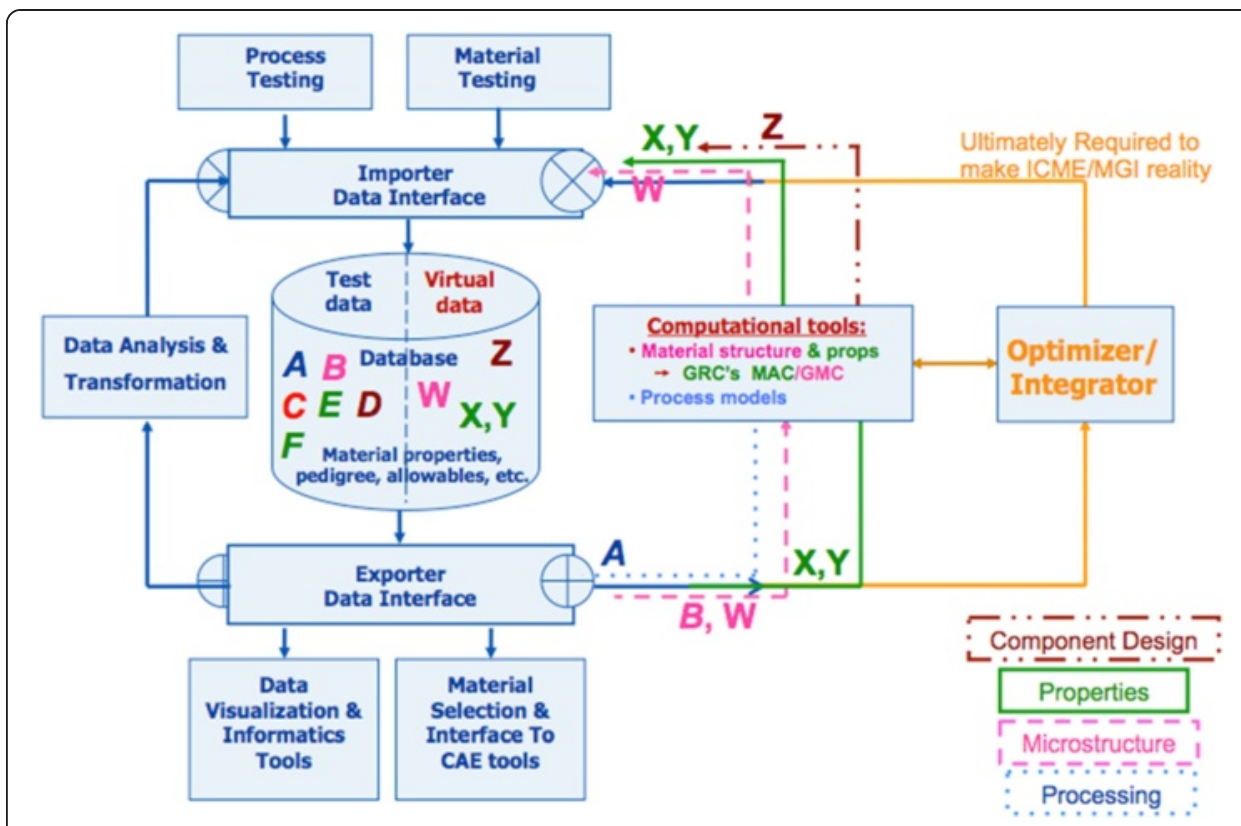

Figure 3 ICME infrastructure for housing modeling and testing information. Private communications with Dr. David Cebon, Cambridge University and Granta Design Ltd., 2013.

performance criteria (e.g., fatigue life, creep rupture, buckling load) $Z$, which is again stored in the database. Clearly such an information management infrastructure not only enables the capture, analysis, dissemination, and maintenance of various types of data but also facilitates the verification and validation of model output and certification of toolsets at multiple length scales. Also, once all of the input/output protocols are established, it can enable the seamless integration of these toolsets with optimization (e.g., OpenMDAO [7]) algorithms that will provide the final linkage of processing to performance criteria - thus realizing true ICME.

In this paper, our interests lie in identifying the challenges, best practices, and required schema with associated attributes to make the integration of virtual data and test data, described in Figure 3, a reality. Specifically, we will discuss and demonstrate the information management system, based on the Granta MI system, being developed at NASA Glenn Research Center (GRC) for storing not only experimental data (exploratory, characterization, and validation test data, see [4]) but also simulation data (both correlation and predictions) resulting from constitutive modeling activities of both monolithic metals and composite materials. This integration is the first step in our attempt to connect both simulation and experimental data at various scales. Consequently, illustrative emphasis will be placed on the requirements (schema and attributes) for the material/model information management software, rather than on the data contained within the systems. In the 'Materials information management system' section, the challenges, best practices, and required schema are described, while in the 'Micromechanics of composite materials and structures' section, a micromechanics analysis code and multiscale framework under development at NASA GRC are briefly introduced so that in the 'Linkage of experimental and virtual data via Composite Model Table' section the multiscale linkage between experimental and virtual composite data can be discussed. 


\section{Methods}

\section{Materials information management system}

The Material Data Management Consortium (MDMC) has defined the material data life cycle (see Figure 2) in an engineering organization as:
A. Capturing/consolidating materials data;
B. Analyzing materials data;
C. Managing and maintaining the information resource;
D. Deploying and using materials information.

Clearly, this life cycle can be applied similarly to other types of data associated with constitutive models, software tools in general, documentation/reference data, etc. In general, data is captured and consolidated from external sources, legacy databases, as well as internal (possibly proprietary) testing programs. Next, data is analyzed and integrated to create/discover useful information pertinent to the various length scales. The third stage of the data life cycle is the continual maintenance of the whole system (the data and information generated as well as the relationships, or links, between them), with the last, but still crucial, step being the deployment (dissemination) of the right information, to the right people, at the right time, and in the right format. Note that the middle ring of Figure 2 provides additional information regarding the type of data utilized and functions performed during each phase in the data life cycle, while the outermost ring details the individuals most likely responsible for these functions.

To support the various activities throughout the data life cycle, it is preferable to have a single, central source, in which all relevant data is captured and consolidated from 'birth' to 'death' and a variety of software tools are fully integrated (preferably seamless). These tools (as depicted in Figure 3) range from i) data input, ii) reduction/analysis, iii) visualization, iv) reporting, v) process/microstructure/property/performance models (in the case of ICME), vi) material parameter estimation (of both actual and 'virtual' materials), vii) statistical and other analyses to reduce the data to a form usable by designers and analysts - for example, calculation of 'design allowables', viii) product life cycle management (PLM), and ix) structural analysis codes that utilize a central database. Note that the models and tools listed in (iv) and (v) can operate on a variety of different length scales, thus potentially requiring scale-specific attributes. An example of a micromechanics (microstructure/property/performance) analysis code, known as MAC/GMC, that enables both the 'design of' and 'design with' composite materials will be described and illustrated in the 'Micromechanics of composite materials and structures' section of this paper. If the resulting predicted properties (i.e., virtual data) are stored in the database as well, then it is straightforward to validate such methods and models by direct comparison with actual test data. These tools should enable material and structural engineers to input, manage, and utilize information in an efficient, reliable, and user-friendly way as possible. Finally, these tools should also enable enterprise-wide (even worldwide) solution or access.

\section{Capture}

To maximize the impact on the material and structural discipline practitioner and/or researcher, more than just specific predefined (generally accepted) point-wise property 
values/information needs to be captured from both tests and simulations. In fact, it is essential that a best practice software infrastructure i) has the ability to capture a materials fundamental multiaxial response spectrum (under a variety of loading conditions), along with its full pedigree (e.g., chemistry, processing, heat treatment, microstructure, and testing information) for subsequent analysis and modeling; ii) has the ability to capture the application potential of a given material system, be it monolithic, composite, multifunctional, etc.; and iii) enables contextual linkage and association of tacit (or hidden) knowledge (e.g., insight, intuition, skills, experience, and other knowledge that has not been formally shared) within a given organization [3].

\section{Analyzing materials data}

For most organizations, a corporate materials database is a dynamic resource - they want to continually add data and to analyze that data to generate new or updated information. This requires software that can process, manipulate, and perform calculations based upon the data. For example, materials experts need software to process raw materials test data and analyze it in order to create approved design data for wider publication. They must update and refine this information and prepare it for use in specialized applications, such as statistical process control or constitutive-life modeling. Such tools are highly specialized and may be developed in-house, come from academic or commercial collaborators, or be purchased. Table 1 lists some examples.

Whatever the exact nature or source of such software tools, best practice materials information management requires that these tools:

- Be able to be used together so that they combine to offer the range of analyses required by materials scientists and engineers - from single test results, to multiple points, to multiple curves;

- Be fully integrated with the information management system, so that data is extracted directly into the analysis tool and results are saved directly back into the correct locations in the database (see the 'Linkage of experimental and virtual data via Composite Model Table' section);

- Their results be permanently linked to raw input data and the details of the analyses performed, so as to maintain full traceability.

Table 1 Examples of analysis tools needed by materials experts

\begin{tabular}{ll}
\hline $\begin{array}{l}\text { Property estimation } \\
\text { tools }\end{array}$ & Thermo-Calc, CALPHAD, MAC/GMC, etc. \\
$\begin{array}{l}\text { Processing test data } \\
\text { Material selection/ } \\
\text { substitution }\end{array}$ & $\begin{array}{l}\text { Tensile tests, compression tests, creep, fatigue crack growth, E399 fracture toughness, } \\
\text { etc. }\end{array}$ \\
$\begin{array}{l}\text { Deformation Models } \\
\text { CES Selector 2015, MatWeb }\end{array}$ & $\begin{array}{l}\text { Fit test load/stress, total strain, and/or inelastic strain as a function of time at various } \\
\text { constant temperatures (tensile, creep, relaxation, cyclic, step tests, etc.). For example, } \\
\text { elastic, viscoelastic, or generalized viscoelastoplastic models. See refs. [18-21] }\end{array}$ \\
$\begin{array}{l}\text { Stress vs. life curves for stress-controlled cyclic tests using models such as the Basquin } \\
\text { model, the Life power model, the Ramberg-Osgood model. Creep strain vs. time, for } \\
\text { creep and creep rupture: Larson Miller model or Kachanov type continuum damage } \\
\text { mechanics (CDM) model. See refs. [18-21] }\end{array}$ \\
\hline
\end{tabular}




\section{Maintaining materials information}

Establishing a 'gold source' of materials information is not enough, as this source must also be protected, nurtured, and maintained. A number of data management features are critical to this process: i) traceability, ii) access control, iii) version control, and iv) data quality control as discussed in detail in references [6,8]. Perhaps the most important requirement for best practice materials information management is the ability to trace relevant information on the materials beyond their property data. Knowing a material's pedigree information can help users understand and correctly apply the materials in component designs and constructions. It also provides important information (processing, microstructure, etc.) and references required for improving the materials properties or developing new materials. Most importantly, it is irrational to be confident in the use of any data if its pedigree is unknown, as using un-pedigreed data (be it simulation or experimental) involves an extreme risk for safety critical structural applications. While today it is common practice (particularly in research) to use data with questionable pedigrees, it is precisely this background data that is essential to capture, analyze, and maintain if ICME is to become a reality in industrial applications. Consequently, the design of the data schema becomes the major issue in ensuring traceability. Note that, to enable both high traceability and high scalability, separating the individual data categories and connecting them with adequate links becomes an essential attribute of any fit-for-purpose information management system. For example, raw, statistical, and design data are considered to be the core data categories, while pedigree, microstructure, testing, application, in-service environment and exposure, and reference data are normally deemed background information.

NASA GRC's Granta $\mathrm{MI}^{\odot}$ installation, illustrated in Figure 4, is an example of such a fit-for-purpose information management system, wherein NASA GRC's data schema (see Figure 5), an extension of the MDMC data schema, has been specifically designed to enable ICME activities. For example, the microstructure information category (table) (see Table 2 for its details of contents) is separated from other material pedigree tables, thus enabling one to go directly to this table and quickly locate typical microstructural images (see Figure 6), and then trace backwards through the links to the other associated material pedigree tables, raw test results, and processing history that produced the specific microstructure. Microscopy information, however, which is associated with changes during testing (due to either mechanical or thermal loading) or subsequent to testing (e.g., failure surface analysis), is typically specimen-specific and thus is stored in the specific specimen record located within the various Test Data tables.

Likewise, to enable scale-specific experimental and model simulation (virtual) data to coexist in the same database, tables associated with 'model pedigree' information (e.g., Deformation, Damage, and Composite) have also been included in the data schema (see Figure 5). Virtual data is an outcome from running some form of model/analysis software tool. For example, in the case of mechanics of materials, this can be as straightforward as exercising a given constitutive model (the simplest being isotropic Hooke's law, which involves only two parameters (e.g., Young's modulus and Poisson's ratio) or as complex as a general nonlinear finite element analysis of a complex structural component resulting in complex response spectrums. In either case, it is essential to understand/record the fundamental assumptions (material system, material anisotropy, linear and nonlinear behavior, boundary conditions, etc.), pertinent model 


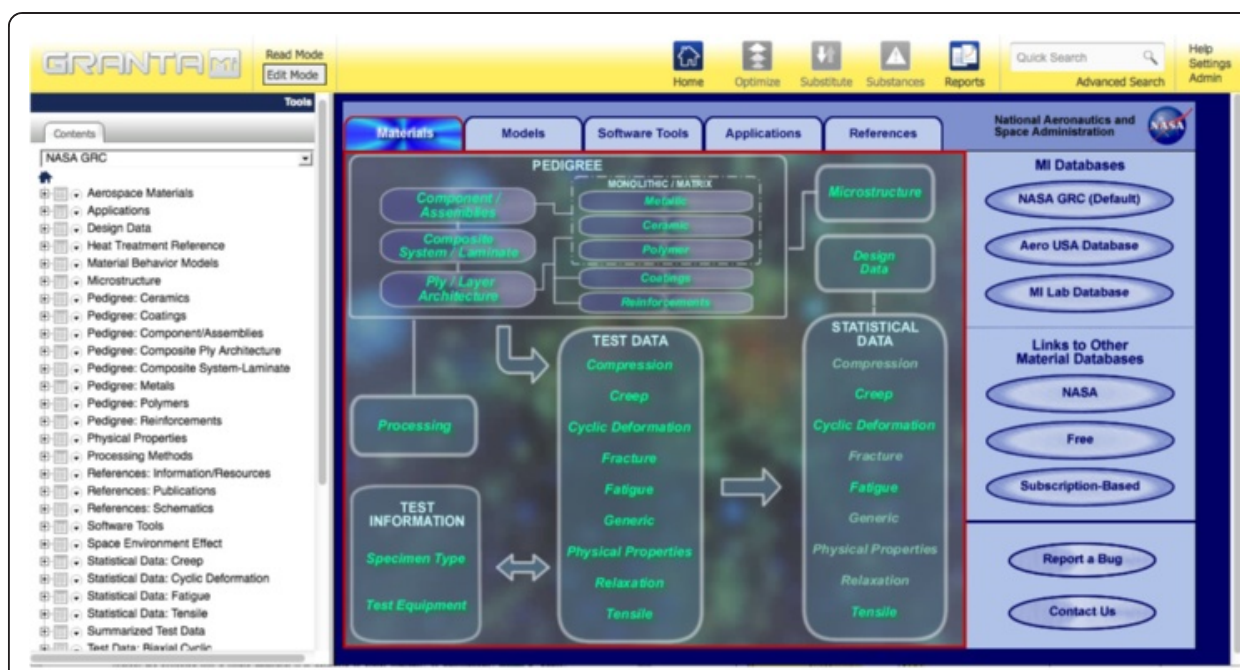

a) Material tab

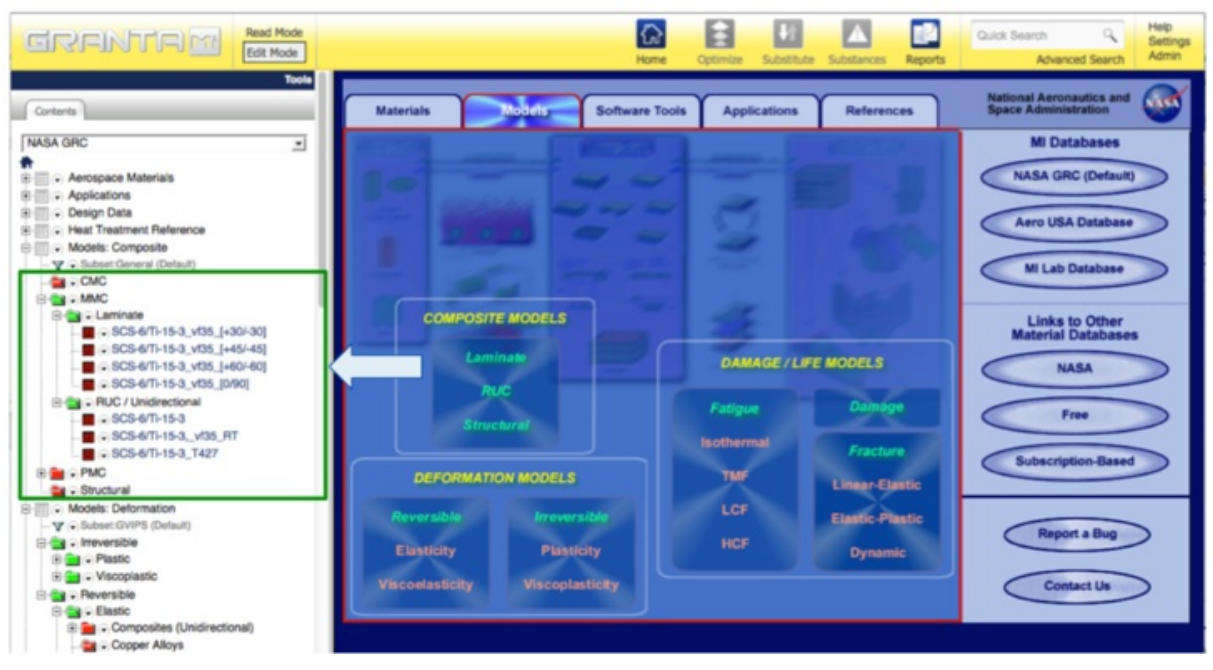

b) Model tab (Composite)

Figure 4 NASA GRC's customization of GRANTA MI materials information management system.

parameters, loading conditions, etc., along with the resulting simulation data itself, in order to properly connect experimental data with simulation data. One might ask, 'Why should I store the resulting simulation data?' The benefits of storing simulation data along with their pedigree information are fourfold: 1) it allows immediate comparison between experiment and simulation, thus enabling an assessment of the accuracy of both the correlation ability and/or predictive ability of the model, 2) it enables periodic re-assessment of the model's accuracy as the experimental data set grows, thus indicating when the model's characterization needs to be updated, 3) it provides future generations with benchmark curves to confirm the version of the model being used or to verify its re-implementation by someone else, and 4) it allows complete traceability, from model version to experimental data used for correlation. Any researcher or analyst who has attempted to reproduce the modeling results of a coworker, or even their own modeling results after a number of years, can attest to the value of storing and tracking simulation data and pedigree. 


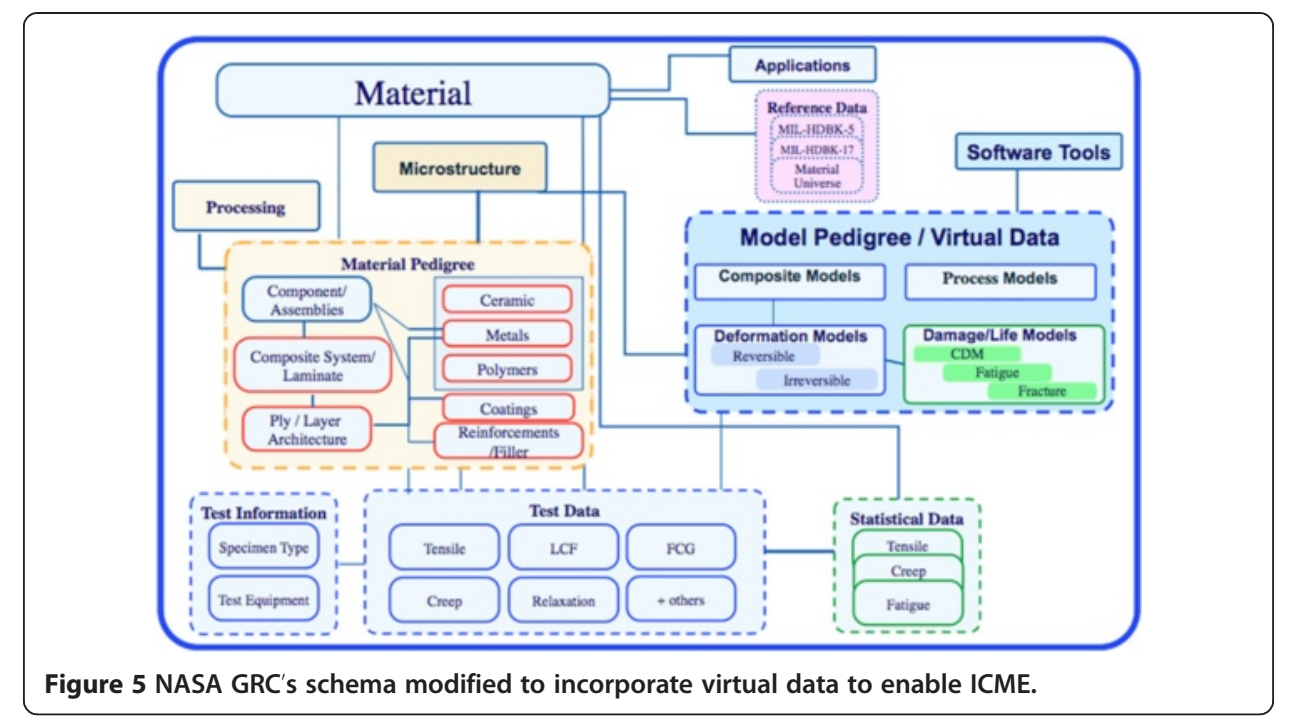

Obviously, ICME involves a wide variety of models (e.g., process models, internal structure models, and constitutive models) as indicated in Table 1 and thus necessitates a versatile schema. In Arnold et al. [6], the specifics of the schema (i.e., required attributes) and the format (e.g., attribute type and record layout) for best storing such information were discussed in detail for storing information limited to monolithic and composite material coupon level data. In the case of monolithic materials (e.g., fiber and matrix), three tables and their associated attributes were defined to enable the complete data life cycle to be handled; these are the following: Deformation Model Table, Damage/Life Model Table, and Software Tools Table (see Figure 5). Whereas, in the case of composite materials, one must think more broadly as multiple length scales can be involved depending upon the approach taken (i.e., macromechanics or micromechanics) to define the material's 'constitutive model.' Consequently, the additional meso- or macroscale above the constituent scale (e.g., that associated with monolithic material) necessitates the introduction of a fourth table, the Composite Table (discussed in detail in the 'Linkage of experimental and virtual data via Composite Model Table' section). Clearly, extension to other scales (e.g., atomistic, processing, microstructure modeling, structural) may require either the addition of new tables with appropriate attributes to the model pedigree group within Figure 5 (e.g., Process Model Table) or new scale-specific attributes to represent each new scale considered. Clearly, the present schema (with its assembly of model pedigree tables) not only allows model information and model parameters to be stored in a location that is easily accessible by FEA or other analysis codes through some type of interface software (e.g., Materials Gateway ${ }^{\circ}$ ) but also stores any associated simulation data necessary to assist in the evaluation, validation/certification, and utilization of these models.

\section{Micromechanics of composite materials and structures}

In its broadest context, a composite is anything comprised of two or more entities with a recognizable interface (i.e., distinct internal boundaries) between them. If these internal boundaries are ignored, continuum mechanics can be used to model composite materials as pseudo-homogenous, anisotropic materials with directionally dependent 'effective,' 'homogenized,' or 'smeared' material properties. Micromechanics, on the 
Table 2 Attributes for microstructure description

\begin{tabular}{|c|c|c|}
\hline \multirow{2}{*}{$\begin{array}{l}\text { Attributes } \\
\text { General }\end{array}$} & \multicolumn{2}{|l|}{ Meta-attributes } \\
\hline & Sizes & Phase compositions \\
\hline Specimen ID & Grain size, measured & Percent \\
\hline Pedigree ID & Standard deviation, ASTM number & Size \\
\hline Disk ID & As-large-as grain size, ASTM number & Photomicrographs \\
\hline Location in disk & Number & Description \\
\hline Relative quench rate & Major axis: average & Etchant \\
\hline Relative stress relief time & Major axis: standard deviation & Image magnification \\
\hline Centroid location & Major axis: range & Image width \\
\hline Centroid - $r$ & Minor axis: average & Image height \\
\hline Centroid - $z$ & Minor axis: standard deviation & Date photo taken \\
\hline Centroid - $\Theta$ & Minor axis: range & Photographer \\
\hline Cutup diagrams & Feret diameter: average & RVE (embedded file) \\
\hline Cutup diagram pictures & Feret diameter: standard deviation & RVE (link to file) \\
\hline Microstructure & Feret diameter: range & Distance from centroid $-x$ \\
\hline Graphic & Aspect ratio: average & Distance from centroid $-y$ \\
\hline Primary $\gamma^{\prime}$, area fraction & Aspect ratio: standard deviation & Distance from centroid - $z$ \\
\hline Secondary $\gamma^{\prime}$, area fraction & Aspect ratio: range & Microscopy technique \\
\hline Tertiary $\gamma^{\prime}$, area fraction & Compactness: average & RVE (representative photo) \\
\hline \multirow[t]{2}{*}{ Minor phases in matrix } & Compactness: standard deviation & Distance from centroid $-z$ \\
\hline & Compactness: range & \\
\hline Minor phases at grain boundaries & Shape factor: average & Microscopy technique \\
\hline Histogram: major axis & Shape factor: standard deviation & RVE (representative photo) \\
\hline Histogram: minor axis & Shape factor: range & \\
\hline \multicolumn{3}{|l|}{ Histogram: Feret diameter } \\
\hline \multicolumn{3}{|l|}{ Mean grain size, ASTM number } \\
\hline \multicolumn{3}{|l|}{ As-large-as grain size, ASTM number } \\
\hline \multicolumn{3}{|l|}{ Grain size, measured } \\
\hline \multicolumn{3}{|l|}{ Standard deviation, ASTM number } \\
\hline \multicolumn{3}{|l|}{ Histogram: aspect ratio } \\
\hline \multicolumn{3}{|l|}{ Histogram: compactness } \\
\hline \multicolumn{3}{|l|}{ Histogram: shape factor } \\
\hline \multicolumn{3}{|l|}{ Supporting graphics } \\
\hline \multicolumn{3}{|l|}{ Micrographs } \\
\hline \multicolumn{3}{|l|}{ Photomicrographs } \\
\hline \multicolumn{3}{|l|}{ Other phases } \\
\hline \multicolumn{3}{|l|}{ Boundary minor phase composition } \\
\hline Matrix minor phase composition & & \\
\hline
\end{tabular}

other hand, attempts to account for the internal boundaries within a composite material and capture the effects of the composite's internal arrangement. In micromechanics, the individual materials (typically referred to as constituents or phases) that make up a composite are each treated as continua via continuum mechanics models, with their individual properties and arrangement dictating the overall behavior of the composite material. Over the past two decades, NASA GRC has been developing the ImMAC suite of tools for 


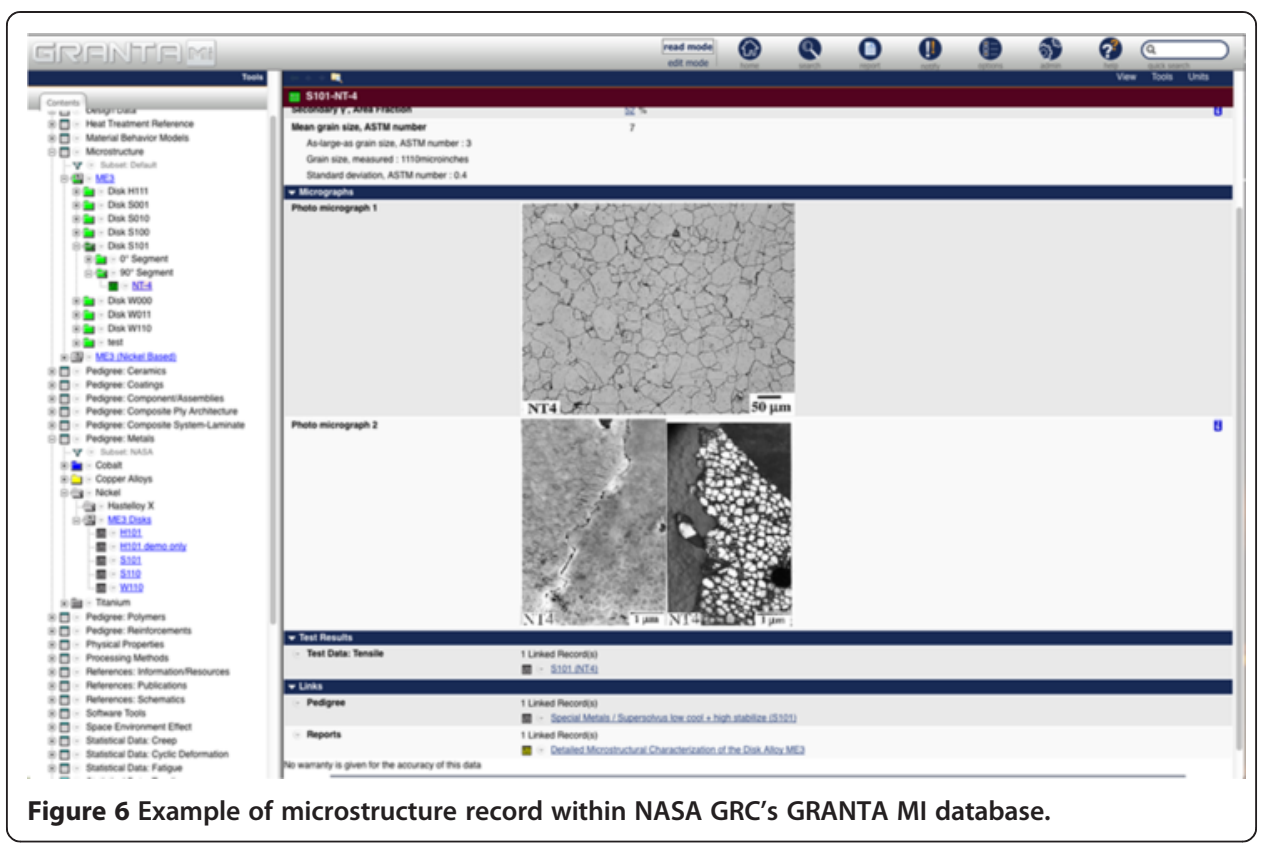

analyzing continuous, discontinuous, woven, and smart (piezo-electromagnetic) composite materials and/or structures composed of such materials. MAC/GMC (a comprehensive and versatile stand-alone micromechanics analysis computer code), HyperMAC (the coupling of MAC/GMC micromechanics with the commercial structural sizing software known as HyperSizer [9]), MSGMC (the recursive coupling of micromechanics with micromechanics, for woven composites), and FEAMAC (the coupling of MAC/GMC micromechanics with the commercial finite element code, Abaqus [10]) make up this suite. At the core of these various tools is the well-known method of cells family of micromechanics theories (e.g., method of cells (MOC), generalized method of cells (GMC), and high-fidelity generalized method of cells (HFGMC)) developed by Aboudi and co-workers [11]. These methods provide semi-closed form solutions for determining global anisotropic composite properties in terms of the constituent material properties and arrangement, while also providing the full three-dimensional stresses and strains in each of the constituent subcells. For a detailed, comprehensive discussion on modeling of composite materials, the reader is referred to the book entitled Micromechanics of Composite Materials: A Generalized Multiscale Analysis Approach [11]. Micromechanics-based analysis lends itself to ICME in that it links the processing and microstructure of the material directly to the resulting properties and performance of the material/structure, thereby enabling the practitioner to not only 'design with' the material but also concurrently 'design the' material. Consequently, developing a database schema capable of handling a micromechanics approach enables demonstration of an ICME capable (multiscale) framework for composite materials.

\section{The generalized method of cells}

It is assumed that a continuously reinforced composite microstructure can be represented as a collection of doubly periodic repeating unit cells (RUCs) containing an arbitrary number of constituents, as shown in Figure 7. The RUC (indicated by a dashed 


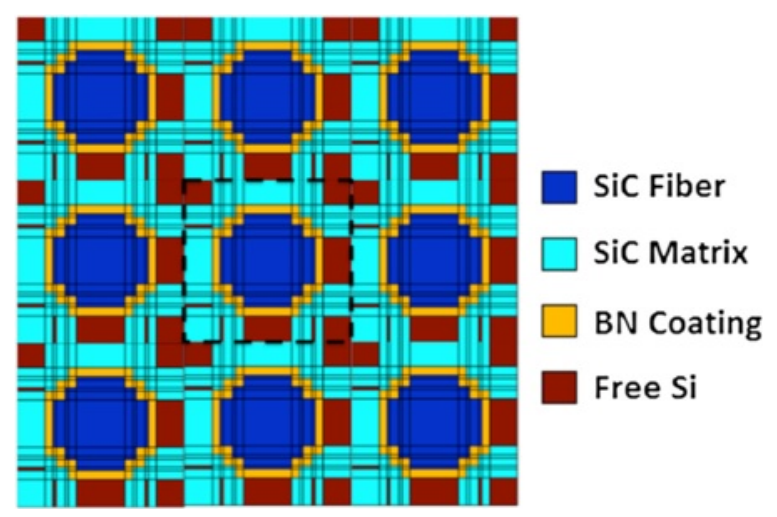

Figure 7 Representation of the doubly periodic microstructure of a CMC composite material.

line in Figure 7) is then discretized into $N_{\beta} \times N_{\gamma}$ rectangular subcells (in the case of doubly periodic generalized method of cells (GMC)), as exhibited in Figure 8. Note that triply periodic microstructures (e.g., particulate-reinforced or 3D woven composites), although not discussed here, can also be easily represented as well. Each of these subcells is occupied by one of the constituent materials (e.g., $\mathrm{SiC}$ fiber, $\mathrm{BN}$ coating, $\mathrm{SiC}$ matrix, and free $\mathrm{Si}$ in the case of $\mathrm{SiC} / \mathrm{SiC}$ composites). The number of subcells and the number of materials are completely general. In GMC, a first-order displacement field within the subcells is assumed, and displacement and traction continuity conditions are enforced in an average, integral sense at the subcell interfaces of a discretized RUC. These continuity conditions are used to formulate a set of semi-analytical linear algebraic equations that are solved for the local strains in subcell $(\beta \gamma)$ in terms of globally applied strains or stresses. Then, local constitutive laws can be utilized to obtain the local stresses in subcell $(\beta \gamma)$ :

$$
\begin{aligned}
& \boldsymbol{\varepsilon}^{(\beta \gamma)}=\mathbf{A}^{(\beta \gamma)} \overline{\boldsymbol{\varepsilon}}+\mathbf{D}^{(\beta \gamma)}\left(\boldsymbol{\varepsilon}_{\mathrm{s}}^{\mathrm{I}}+\boldsymbol{\varepsilon}_{\mathrm{s}}^{\mathrm{T}}\right) \\
& \boldsymbol{\sigma}^{(\beta \gamma)}=\mathbf{C}^{(\beta \gamma)}\left\{\mathbf{A}^{(\beta \gamma)} \overline{\boldsymbol{\varepsilon}}+\mathbf{D}^{(\beta \gamma)}\left(\boldsymbol{\varepsilon}_{\mathrm{s}}^{\mathrm{I}}+\boldsymbol{\varepsilon}_{\mathrm{s}}^{\mathrm{T}}\right)-\left(\boldsymbol{\varepsilon}^{\mathrm{I}(\beta \gamma)}+\boldsymbol{\varepsilon}^{\mathrm{T}(\beta \gamma)}\right)\right\}
\end{aligned}
$$

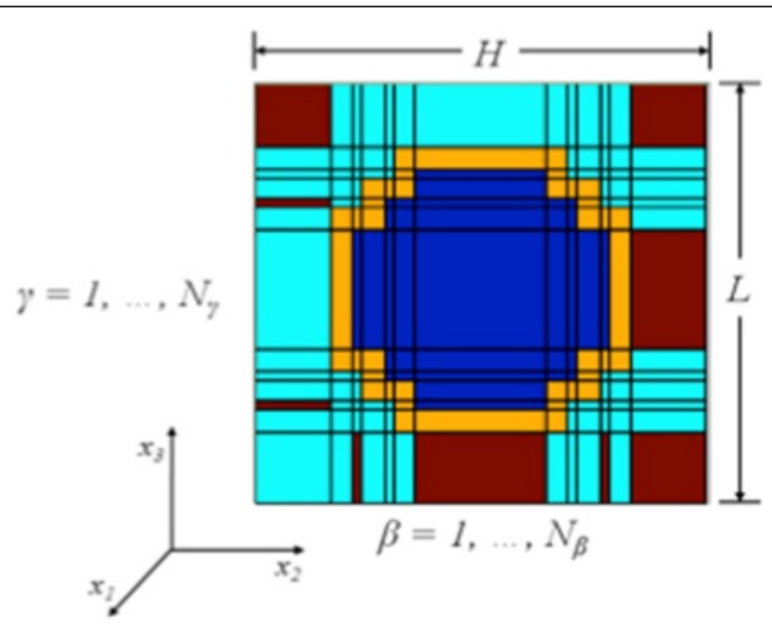

Figure 8 Discretization of a doubly periodic RUC. 
where $\boldsymbol{\sigma}$ is the stress tensor, $\mathbf{C}$ is the stiffness tensor, and $\boldsymbol{\varepsilon}, \boldsymbol{\varepsilon}^{I}$, and $\boldsymbol{\varepsilon}^{T}$ are the total, inelastic, and thermal strain tensors, respectively, $\boldsymbol{\varepsilon}_{\mathrm{s}}^{\mathrm{I}}$ and $\boldsymbol{\varepsilon}_{s}^{T}$ are 6 by $N_{\beta} N_{\gamma}$ matrices containing all components of the inelastic and thermal strains, respectively, of every subcell (appropriately ordered), $\mathbf{A}^{(\beta \gamma)}$ is the strain concentration tensor, and $\mathbf{D}^{(\beta \gamma)}$ is the thermo-inelastic strain concentration tensor. Then, the generalized constitutive law for the effective, homogenized composite can be formulated as:

$$
\overline{\boldsymbol{\sigma}}=\mathbf{C}^{*}\left(\overline{\boldsymbol{\varepsilon}}-\overline{\boldsymbol{\varepsilon}}^{\mathrm{I}}-\overline{\boldsymbol{\varepsilon}}^{\mathrm{T}}\right)
$$

where the effective stiffness tensor, $\mathbf{C}^{*}$, effective inelastic strains, $\overline{\boldsymbol{\varepsilon}}^{\mathrm{I}}$, and effective thermal strains, $\overline{\boldsymbol{\varepsilon}}^{\mathrm{T}}$, are given by:

$$
\begin{aligned}
& \mathbf{C}^{*}=\frac{1}{h l} \sum_{\beta=1}^{N_{\beta}} \sum_{\gamma=1}^{N_{\gamma}} h_{\beta} l_{\gamma} \mathbf{C}^{(\beta \gamma)} \mathbf{A}^{(\beta \gamma)} \\
& \overline{\boldsymbol{\varepsilon}}^{\mathrm{I}}=-\frac{\mathbf{C}^{*-1}}{h l} \sum_{\beta=1}^{N_{\beta}} \sum_{\gamma=1}^{N_{\gamma}} h_{\beta} l_{\gamma} \mathbf{C}^{(\beta \gamma)}\left(\mathbf{D}^{(\beta \gamma)} \boldsymbol{\varepsilon}_{\mathrm{s}}^{\mathrm{I}}-\mathbf{\varepsilon}^{\mathrm{I}(\beta \gamma)}\right) \\
& \overline{\boldsymbol{\varepsilon}}^{\mathrm{T}}=-\frac{\mathbf{C}^{*-1}}{h l} \sum_{\beta=1}^{N_{\beta}} \sum_{\gamma=1}^{N_{\gamma}} h_{\beta} l_{\gamma} \mathbf{C}^{(\beta \gamma)}\left(\mathbf{D}^{(\beta \gamma)} \boldsymbol{\varepsilon}_{\mathrm{s}}^{\mathrm{T}}-\boldsymbol{\varepsilon}^{\mathrm{T}(\beta \gamma)}\right)
\end{aligned}
$$

$h_{\beta}$ and $l_{\gamma}$ are the dimensions of the subcells, $h$ and $l$ are the dimensions of the RUC, and $\overline{\boldsymbol{\sigma}}$ and $\overline{\boldsymbol{\varepsilon}}$ are the effective (homogenized) stress and strain tensors, respectively. Extensive details regarding this derivation can be found in Aboudi et al. [11].

\section{Results and discussion}

To illustrate the potential utility of micromechanics for ICME of composite materials, the influence of residual stresses and subsequent post-heat treatment on the laminate response of a $\left[0^{\circ} / 90^{\circ}\right]_{\mathrm{s}} \mathrm{SiC} / \mathrm{SiC} \mathrm{CMC} \mathrm{composite} \mathrm{laminate} \mathrm{will} \mathrm{be} \mathrm{examined.} \mathrm{The} \mathrm{consi-}$ tuents present within the $\mathrm{SiC} / \mathrm{SiC}$ RUC include a $\mathrm{SiC}$ matrix, $\mathrm{SiC}$ fiber, $\mathrm{BN}$ coating, and free $\mathrm{Si}$ inclusions. Here, the effect of creep of the constituents (wherein it was assumed that the creep of the $\mathrm{SiC}$ fiber is less than the creep of the $\mathrm{SiC}$ matrix which is less than the creep of the free $\mathrm{Si}$ for all temperatures) is accounted for by assuming a simple Norton-Baily power law, $\dot{\varepsilon}^{I}=A \sigma^{n}$, for the fiber, matrix, and free $\mathrm{Si}$ inclusions within the matrix. Note that the BN coating is assumed to be elastic, very compliant, and nondamaging in this illustration.

The qualitative effect of including residual stress effects resulting from manufacturing on the proportional limit stress (PLS) and strain to failure $\left(\varepsilon_{\mathrm{f}}\right)$ of a typical simulated tensile test performed at room temperature is shown in Figure 9. Applying a subsequent post-heat treatment $(\mathrm{HT})$ at different temperatures and for different durations shows that the PLS and strain to failure at room temperature can be impacted, as illustrated in Figure 10. Note that the degree of impact (i.e., the amount of redistribution of residual stress) is a function of stress, time, temperature, and microstructure. Clearly, the increase in PLS and decrease in strain to failure, resulting from residual stress as shown in Figure 9, is diminished as the hold time and temperature are increased (see Figure 10). Furthermore, a macromechanics modeling approach could not predict such post-HT tensile behavior, since it is due to internal stress 


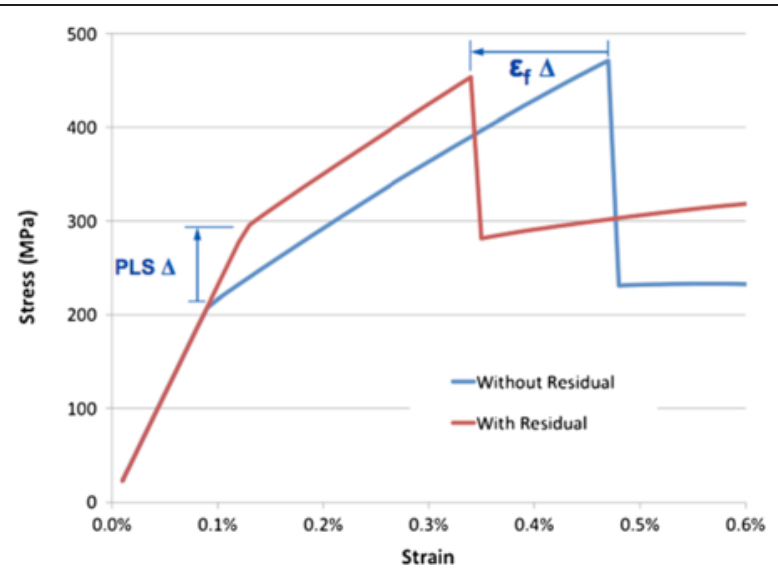

Figure 9 Qualitative influence of residual stress on the tensile response of a $\left[0^{\circ} / 90^{\circ}\right]_{\mathrm{s}} \mathrm{SiC} / \mathrm{SiC}$ CMC laminate.

redistribution within the composite material itself that occurs during (globally) stress-free manufacturing and post-processing conditions. Such behavior has been observed by Bhatt experimentally (private communications). Consequently, micromechanics provides a seamless link between the two non-exclusive viewpoints of designing 'with' the material and designing 'the' material, thereby enabling ICME of composite materials.

For ICME, it is necessary to link the subscale effects to structural performance. As such, a synergistic multiscale framework (which executes concurrent multiscaling in time, but sequential multiscaling in space [12]) has been constructed to simulate the nonlinear response of fiber-reinforced composite structures by modeling the fibermatrix architecture as an RUC at the microscale using GMC and coupling the microscale to the lamina/laminate level (macroscale) finite element model (FEM). The commercial finite element software, Abaqus [10], is used as the FEM platform, and the MAC/GMC core micromechanics software $[13,14]$ is used to perform microscale calculations. The scales are linked using the FEAMAC software implementation [15], which utilizes various Abaqus/Standard user subroutines. A schematic displaying a

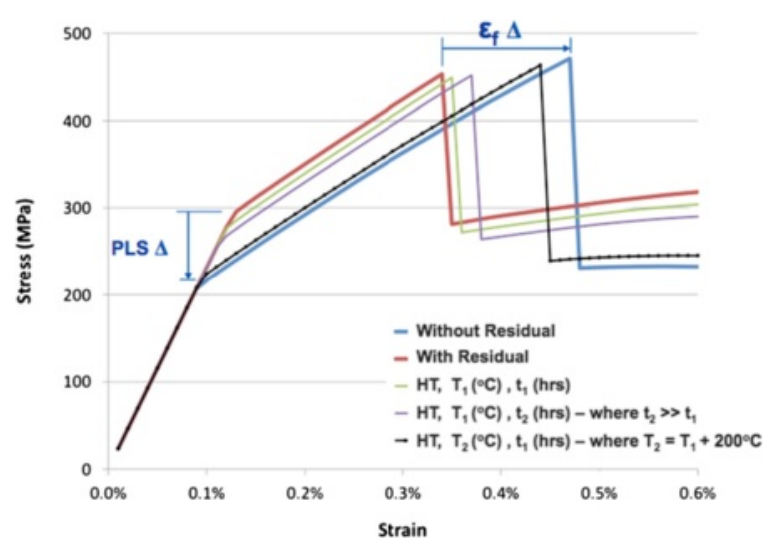

Figure 10 Qualitative effect of heat treatment on tensile response of a $\left[0^{\circ} / 90^{\circ}\right]_{\mathrm{s}} \mathrm{SiC} / \mathrm{SiC}$ CMC laminate. 
typical multiscale model using FEAMAC is displayed in Figure 11. The strains at the FEM integration point are applied to the RUC, and the local subcell fields are determined using GMC (this process is referred to as localization). If the subcell material behavior is nonlinear, the local stresses and strains are used to calculate the local stiffnesses, inelastic strains, thermal strains, and/or state variables via the local constituent constitutive laws. Homogenization of the RUC is then performed to obtain the global (effective) stiffnesses, inelastic strains, thermal strains, and/or state variables. The global stresses at the integration point are then calculated using these global, homogenized fields, and the effects of any nonlinear subscale phenomena are introduced into the macroscale through changes in the integration point stress state and stiffness. The global stresses, material Jacobian, and updated state variables at each FEM integration point are then supplied to Abaqus through the user material UMAT subroutine. For complete details on the FEAMAC implementation, the reader is referred to Bednarcyk and Arnold [15] and Aboudi et al. [11].

With the ability to link the GMC micromechanics model, which accounts for processing and microstructure while predicting properties (as discussed in the previous section), with a structural FEM, which simulates performance, the full range of ICMErelated scales depicted in Figure 3 has been captured. To briefly illustrate the connection of all aspects of the multiscale (constituent, meso/composite, structural) problems just outlined in Figures 7, 8, 9, 10, and 11 (i.e., material, microstructure, and model pedigree along with test results and software tool description), a repeat of Figure 5, but now with specific names of potential records, is indicated in their pertinent tables within the proposed schema (see Figure 12). Clearly, this is a very high level overview illustration, yet the hope is that it elucidates how one might connect simulation and experimental results and their associated pedigrees together in a single database. Note that the exact location of the simulation results of the structural analysis (i.e., stiffened panel) has yet to be finalized as they could go in a model pedigree table, the application table, or in a PLM system external to the Granta MI database. More specific details regarding layout and associated attributes within the composite and software tables are given in the next section.

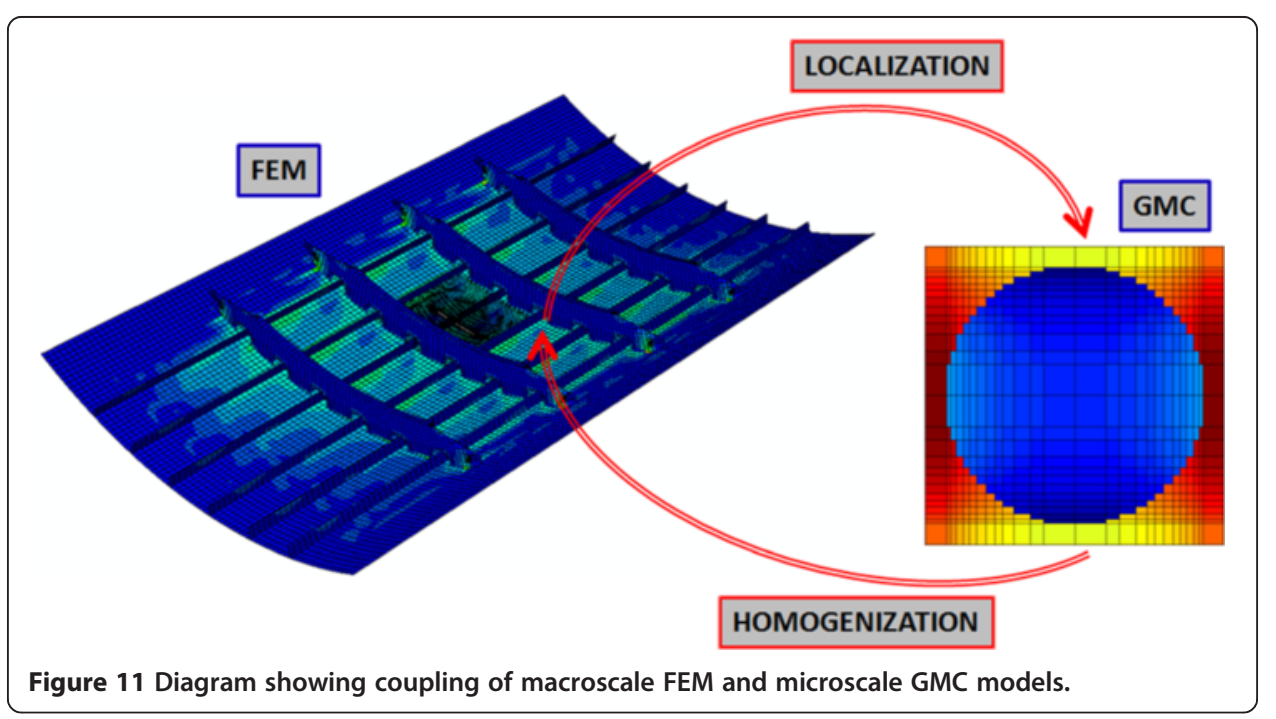




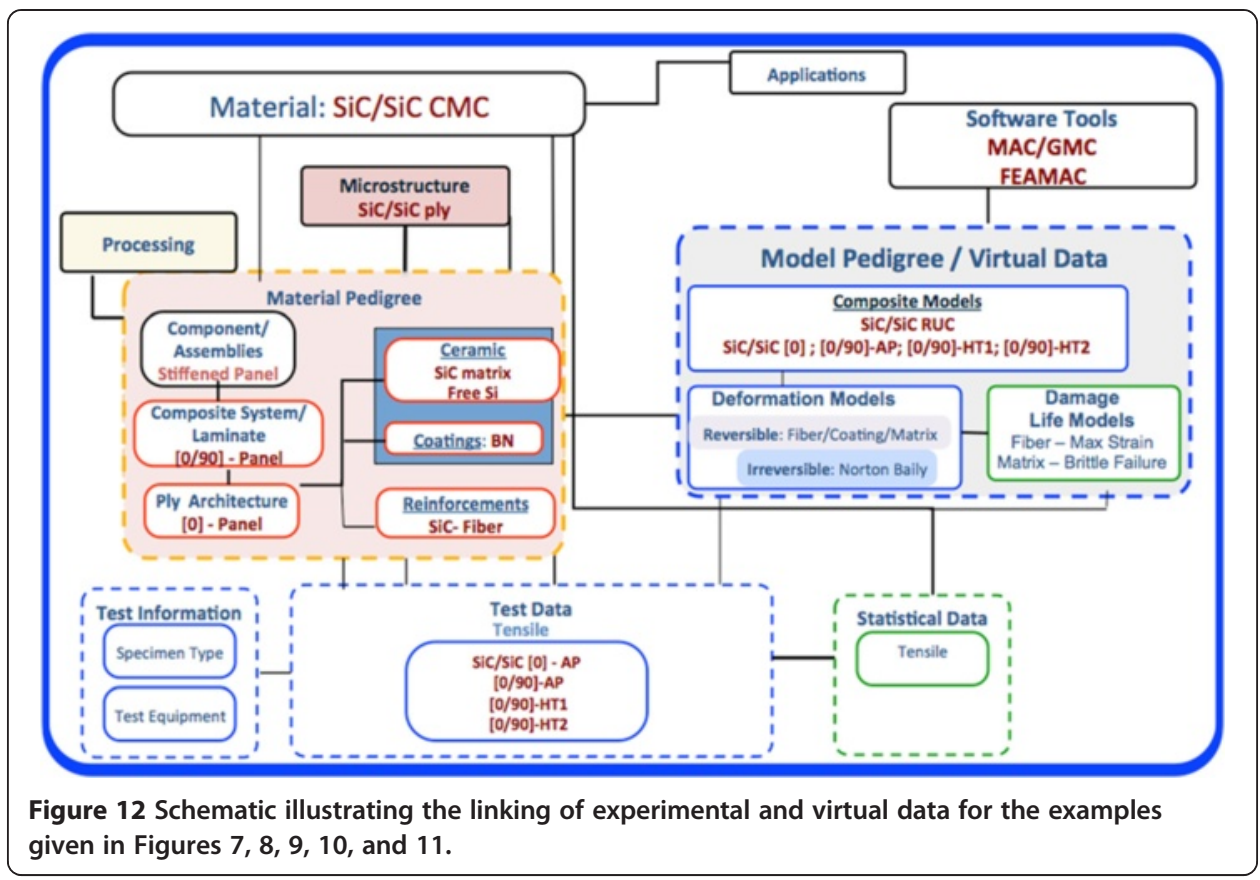

Linkage of experimental and virtual data via Composite Model Table

As stated previously, foundational to any ICME endeavor (albeit research or product design) is a robust information management system in which both experimental and model simulation (virtual) data coexist, preferably in a single database, at various levels of scale. Just as in the case of experimental data, capturing the pedigree of the material tested is an essential step to enable proper interpretation of results, so too is tracking the pedigree of any simulation (virtual) data entered into the database. Consequently, following the same principles invoked to create material pedigree tables and their linkage to experimental data, a similar thought process was followed to create a set of model pedigree tables that will house all information related to simulations performed at various levels of scale.

The Composite Model Table, as depicted previously in Figure 5, consists of ten basic sections: 'Project Information,' 'Material Description,' 'General Modeling Information,' 'Micromechanics Modeling Information,' 'Laminate Level Modeling Information,' 'Volume Fractions,' 'Multiscale Modeling Information,' 'Composite Test Data Used for Characterization/Validation,' 'Simulation Response', and 'References' (see Table 3). The first section is associated with the Project Information. The second, Material Description, section, is where the model record is connected to the specific material (or system) that the model is attempting to represent. This is accomplished by linking the material pedigree (via the various attributes in this section (see Table 3) and specifically the material pedigree record link) to the model idealization information contained in the current Model Table record.

The model description section gives the general features of the model, yet in this table, there is no explicit section entitled 'Characterization Information/Parameter Estimation Method' as exists in the Deformation Model Table (described in Arnold et al. [6]). The reason is that this information would be contained in the Deformation Model Table associated with the various constituent materials constitutive models, in the case 
Table 3 Layout and attributes for Composite Model Table

Attributes

Type

Project Information

Performing Organization

Project Name/Funding Source

STXT

Point of Contact (POC)

STXT

Material Description

Material

STXT

Material Class

DCT

Commercial Name

STXT

Specific Name

STXT

Material Pedigree Record

Link

Batch Number

STXT

Material Notes

LTXT

General Modeling Information

Model ID

Characterization/Analysis Date

DAT

Temperature

PNT

Temperature Range

RNG

Assumptions

Micromechanics Modeling Information

Micromechanics Method

DCT

Micromechanics Analysis Tool

STXT

Micromechanics Tool Information

Link

Micomechanics Input File

Fil

No. of Constituents

INT

RUC/RVE Constitutive Description

TABL

RUC/RVE Image

PIC

Fiber Packing Arrangement

DCT

Effective Thermo-Elastic Composite Properties

TABL

Micromechanics Notes

Laminate Level Modeling Information

Laminate Name

Laminate Specification

STXT

Architecture Type

DCT

Laminate Pattern

DCT

Laminate Thickness

PNT

Ply Thickness (avg)

PNT

No. of Plies

INT

Laminate Definition

TABL

Laminate Analysis Tool

STXT

Laminate Analysis Tool Information

STXT

Composite Laminate Analysis Input File

FIL

Laminate Notes 
Table 3 Layout and attributes for Composite Model Table (Continued)

\begin{tabular}{|c|c|}
\hline Laminate Extensional Stiffness Matrix (A) & TABL \\
\hline Laminate Coupling Stiffness Matrix (B) & $\mathrm{TABL}$ \\
\hline Laminate Bending Stiffness Matrix (D) & $\mathrm{TABL}$ \\
\hline \multicolumn{2}{|l|}{ Volume Fractions } \\
\hline Total Matrix Volume Fraction & PNT \\
\hline Total Reinforcement Volume Fraction & PNT \\
\hline Total Void/Porosity Volume Fraction & PNT \\
\hline \multicolumn{2}{|l|}{ Multiscale Modeling Information } \\
\hline Multiscale Analysis Tool & $\mathrm{DCT}$ \\
\hline Multiscale Analysis Tool Information & Links \\
\hline Multiscale Analyses Input File & FIL \\
\hline Multiscale Modeling Notes & LTXT \\
\hline \multicolumn{2}{|l|}{ Composite Test Data Used for Characterization/Validation } \\
\hline Tensile Test Data (Linked Records location in layout) & Links \\
\hline Creep Test Data (Linked Records location in layout) & Links \\
\hline Relaxation Test Data (Linked Records location in layout) & Links \\
\hline Cyclic Test Data (Linked Records location in layout) & Links \\
\hline Generic Test Data (Linked Records location in layout) & Links \\
\hline \multicolumn{2}{|l|}{ Simulation Response } \\
\hline Stress vs. Strain Response (11 axis) & FDA \\
\hline Stress vs. Strain Response (22 axis) & FDA \\
\hline Stress vs. Strain Response (33 axis) & FDA \\
\hline Stress (11 axis) vs. Time & FDA \\
\hline Stress (22 axis) vs. Time & FDA \\
\hline Stress (33 axis) vs. Time & FDA \\
\hline Total Strain (11 axis) vs. Time & FDA \\
\hline Total Strain (22 axis) vs. Time & FDA \\
\hline Total Strain (33 axis) vs. Time & FDA \\
\hline Shear Stress vs. Shear Strain Response (12 axis) & FDA \\
\hline Shear Stress vs. Shear Strain Response (13 axis) & FDA \\
\hline Shear Stress vs. Shear Strain Response (23 axis) & FDA \\
\hline Shear Stress (12 axis) vs. Time & FDA \\
\hline Shear Stress (13 axis) vs. Time & FDA \\
\hline Shear Stress (23 axis) vs. Time & FDA \\
\hline Total Shear Strain (12 axis) vs. Time & FDA \\
\hline Total Shear Strain (13 axis) vs. Time & FDA \\
\hline Total Shear Strain (23 axis) vs. Time & FDA \\
\hline Force Resultant vs. Midplane normal strain (xx-axis) & FDA \\
\hline Force Resultant vs. Midplane normal strain (yy-axis) & FDA \\
\hline Force Resultant vs. Midplane normal strain (xy-axis) & FDA \\
\hline Moment Resultant vs. Midplane curvature (xx-axis) & FDA \\
\hline Moment Resultant vs. Midplane curvature (yy-axis) & FDA \\
\hline Moment Resultant vs. Midplane curvature (xy-axis) & FDA \\
\hline
\end{tabular}


Table 3 Layout and attributes for Composite Model Table (Continued)

\begin{tabular}{l} 
References \\
General Modeling Notes \\
Model References \\
\hline DCT, discrete text (specified choices); FDA, functional data attribute (with associated parameters); FIL, allows the \\
association of any file type to a given record; INT, integer value; LOG, logical; LTXT, long text field; PIC, allows association \\
of any image format to a given record; PNT, point value; RNG, range variable; STXT, short text field; TABL, tabular \\
attribute (multiple columns of data - PNT, STXT, DCT, INT, link). Italics are used to assist the reader in locating/connecting \\
specific attributes to discussion in the text and subsequent figures.
\end{tabular}

of a micromechanics approach. Similarly, in the case of a macromechanics approach, the anisotropic model parameters associated with a given unidirectional 'ply' level material would be stored (along with characterization information (e.g., links to the various tests used to obtain these material parameters)) in their corresponding records in the Deformation Model Table as well.

However, three new sections, specific to composite materials, are present: 'Micromechanics Modeling Information,' 'Laminate Level Modeling Information', and 'Multiscale Modeling Information', with only one of these sections per record being populated - depending upon the type of composite analysis being performed. Note that in each of these sections, not only is the analysis tool (again, uniquely defined in the Software Tool Table shown in Table 4) identified but also the associated input file required to perform the simulations whose results are stored in the Simulation Response section is required. This is necessary because composites typically require more than just a single set of constitutive model parameters in order to reproduce the simulation results (e.g., in the case of micromechanics, geometric and processing information is also required). Note that the attributes 'Software Tool Used' and 'Regression Software Used' in the Deformation Model Table and 'Micromechanics Analysis Tool,' 'Laminate Analysis Tool,' and 'Multiscale Analysis Tool' in the Composite Model Table allow the best practice of only defining information in one location, yet enabling viewing in multiple locations, to be followed as these attributes link the current model record to the Software Tools Table which contains all the pertinent information regarding the specific model/tool being utilized, i.e., its source code and executable - see Table 4 for the associated attributes and layout.

Two new tabular attributes are defined to represent the RUC or representative volume element (RVE) information and the laminate-level information. Figure 13 illustrates both types of tabular attributes, where each column heading represents a parameter associated with the given tabular attribute. Figure 13a provides an example of a unidirectional, 35\% fiber volume fraction, titanium matrix composite (SCS-6/Ti-15-3) represented using the GMC micromechanics approach. Immediately, one sees that two phases are present (fiber and matrix) and that the fiber phase is modeled as an elastic material with its strength being represented by the Curtin fiber breakage model [11]. The matrix phase is modeled as an elastic/plastic material with its fatigue life represented using the anisotropic nonlinear cumulative damage rule - ADEAL [11]. Similarly, the evolving compliant interface (ECI) debond criterion [11] is used between the fiber and matrix phase.

Figure 13b illustrates a fictitious laminate in which a monolithic Ti-15-3 layer is surrounded by a $35 \%$ volume fraction, unidirectional SCS-6/Ti-15-3 ply oriented at +45 on the bottom and -45 on the top. Note that the tabular attribute parameter 'Scale' identifies whether a micromechanics approach (indicated by 'RUC') or macromechanics 
Table 4 Layout and attributes for Software Tools Table

\begin{tabular}{|c|c|}
\hline Attributes & Type \\
\hline \multicolumn{2}{|l|}{ General Description } \\
\hline Tool Name & STXT \\
\hline Version & STXT \\
\hline Description & STXT \\
\hline Component/System Application & STXT \\
\hline Tool Scope & $\mathrm{DCT}$ \\
\hline Method & $\mathrm{DCT}$ \\
\hline Software Required to Execute Code & $\mathrm{DCT}$ \\
\hline Other Software Required to Execute Code & STXT \\
\hline Integration With Other Software & STXT \\
\hline Website & HYP \\
\hline Availability & $\mathrm{DCT}$ \\
\hline Last Update (Year) & $\mathrm{DCT}$ \\
\hline Description Notes & LTXT \\
\hline Classification & STXT \\
\hline Analysis Design & LOG \\
\hline Lifing & LOG \\
\hline Optimization & LOG \\
\hline Thermal/Heat Transfer & LOG \\
\hline Thermodynamics & LOG \\
\hline CFD & LOG \\
\hline Data Analysis & LOG \\
\hline Other Classification & STXT \\
\hline \multicolumn{2}{|l|}{ Domain } \\
\hline Length Scale & $\mathrm{DCT}$ \\
\hline Temporal Scale & $\mathrm{DCT}$ \\
\hline Multiaxiality & $\mathrm{DCT}$ \\
\hline Variables & $\mathrm{DCT}$ \\
\hline Domain Notes & LTXT \\
\hline Material System Applicability & PNT \\
\hline Material Independent & LOG \\
\hline Metallic & LOG \\
\hline Ceramic & LOG \\
\hline Polymer & LOG \\
\hline Composite/Continuous & LOG \\
\hline Composite/Discontinuous & LOG \\
\hline Composite/Woven & LOG \\
\hline Multifunctional & LOG \\
\hline Smart & LOG \\
\hline Nano & LOG \\
\hline Other Material(s) & STXT \\
\hline Material System Notes & LTXT \\
\hline \multicolumn{2}{|l|}{ Material Description } \\
\hline Material Directionality & $\mathrm{DCT}$ \\
\hline
\end{tabular}


Table 4 Layout and attributes for Software Tools Table (Continued)

\begin{tabular}{|c|c|}
\hline Material Scope & DCT \\
\hline Material Response & $\mathrm{DCT}$ \\
\hline Geometric Description & DCT \\
\hline Reversible & LOG \\
\hline Irreversible & LOG \\
\hline Material Description Notes & LTXT \\
\hline \multicolumn{2}{|l|}{ Platform Supported } \\
\hline PC & LOG \\
\hline Mac & LOG \\
\hline \multicolumn{2}{|l|}{ Operating System Supported } \\
\hline Windows 8 & LOG \\
\hline Windows 7 & LOG \\
\hline Windows NT & LOG \\
\hline MacOS & LOG \\
\hline Unix & LOG \\
\hline Linux & LOG \\
\hline Operating System Notes & LTXT \\
\hline \multicolumn{2}{|l|}{ Documentation } \\
\hline Reference Manual & LOG \\
\hline User's Manual & LOG \\
\hline References & LTXT \\
\hline \multicolumn{2}{|l|}{ Verification/Validation Method } \\
\hline Analytical & $\mathrm{DCT}$ \\
\hline Computation & $\mathrm{DCT}$ \\
\hline Experimental & $\mathrm{DCT}$ \\
\hline Verification Notes & LTXT \\
\hline \multicolumn{2}{|c|}{ Software Technology Readiness Level (TRL) } \\
\hline TRL (1-9) & DCT \\
\hline Readiness Notes & LTXT \\
\hline \multicolumn{2}{|l|}{ Availability } \\
\hline Approved for General Release & LOG \\
\hline Security Classification & $\mathrm{DCT}$ \\
\hline Availability Category & $\mathrm{DCT}$ \\
\hline Sensitivity & $\mathrm{DCT}$ \\
\hline Distribution Limitations & DCT \\
\hline Limited Until (month/year) & STXT \\
\hline Point of Contact (POC) & STXT \\
\hline POC's Organization & STXT \\
\hline Availability Notes & LTXT \\
\hline \multicolumn{2}{|l|}{ Source Code } \\
\hline Development Language & DCT \\
\hline Development Language (other) & STXT \\
\hline Source Code Available & LOG \\
\hline Source Code Availability Cat. & DCT \\
\hline Source Code POC & STXT \\
\hline
\end{tabular}


Table 4 Layout and attributes for Software Tools Table (Continued)

\begin{tabular}{lc}
\hline Source Code Location & HYP \\
Source Code Notes & LTXT \\
Ownership Rights & \\
Developer/Performing Org. & STXT \\
Sponsoring Organization & STXT \\
Intellectual Property & DCT \\
Invention Disclosure Filed & LOG \\
NASA Case No. & STXT \\
Distribution Category & DCT \\
Notes & LTXT \\
Program & \\
Project Name/Funding Source & STXT \\
Contract No. & STXT \\
Grant No. & STXT \\
Year Initiated & STXT \\
Software Development Status & DCT \\
Year Completed/Terminated & STXT \\
Project Notes & LTXT \\
Further Information & \\
Software Reports & Links \\
\hline DCT, discrete text (specified choices); FDA, functional data attribute (with associated parameters); HYP, hyperlink; INT, \\
integer value; LOG, logical; LTXT, long text field; PNT, point value; RNG, range variable; STXT, short text field; TABL, \\
tabular attribute (multiple columns of data - PNT, STXT, INT, link).
\end{tabular}

approach (indicated by 'Effective') is being applied to a given layer. In the case of layers 1 and 3, information regarding the modeling of this composite material would be contained in the RUC composite record named 'SCS6/Ti15-3' whose constitutive description is shown in Figure 13a. Therefore, each record referenced can depict a given scale with the interconnection between the constituent scale and the laminate (meso) scale contained within the laminate information tabular attribute.

Next, the 'Simulation Responses' section is where all virtual data is stored. Currently, these functional data attributes (FDAs), e.g., stress vs. strain response (11 axis) and

\begin{tabular}{|c|c|c|c|c|c|}
\hline Phase & Type & Def. Rev. & Def. Irrev. & Damage/Life & Volume Fraction \\
\hline 1 & Fiber & SCS-6 & & Curtin:SCS-6 & 35 \\
\hline 2 & Matrix & Ti-15-3E & InPlas:Ti-15-3 & ADEAL-Ti15-3 & 65 \\
\hline & Debond & & & ECI-SCS6/Ti-15-3 & \\
\hline
\end{tabular}

Note: Type is DCT parameter ( Fiber/Fill/Debond/Interface/Matrix )

a) Example of the RUC/RVE Constitutive Description attribute (Table 3) filled out

\begin{tabular}{|c|c|c|c|c|c|c|c|c|c|}
\hline Ply No. & $\begin{array}{c}\text { Ply } \\
\text { thickness }\end{array}$ & Ply Angle & Scale & RUC Record & Def. Rev. & Def. Irrev. & Damage/Life & Architecture & $\begin{array}{c}\text { Fiber Volume } \\
\text { Fraction }\end{array}$ \\
\hline 1 & 0.04 & -45 & RUC & SCS6/Ti15-3 & & & & Square & 35 \\
\hline 2 & 0.02 & 0 & Effective & & Ti-15-3E & InPlas:Ti-15-3 & ADEAL-Ti15-3 & & \\
\hline 3 & 0.04 & 45 & RUC & SCS6/Ti15-3 & & & & Hexagonal & 35 \\
\hline
\end{tabular}

b) Example of the Laminate Definition attribute (Table 3) filled out

Figure 13 Example of new tabular attributes to describe the composite pedigree. (a) Example of the RUC/RVE Constitutive Description attribute (Table 3) filled out. (b) Example of the Laminate Definition attribute (Table 3) filled out. 
in-plane normal loading (force resultant $(N)$ vs. midplane normal strain $(\varepsilon)$ ) and curvatures (moment resultant $(M)$ vs. midplane curvatures $(\kappa)$ ), have been assigned 13 parameters in all. Ten of these FDA parameters are common to both the Deformation Model Table and Composite Model Table (i.e., specimen ID, test type, loading rate type, loading rate stress magnitude, loading rate strain magnitude, target type, target value, hold duration, simulation classification, and temperature), with four being identified as discrete (i.e., those associated with type and classification), while three are specific to the Composites Model Table - the volume fraction, orientation/layup (this is a short text data type), and resultant specifier (this is a discrete data type, with options: mechanical, inelastic, and thermal). In this way, multiple loading histories can be stored in a single attribute that represents a given graphical plotting space, for example, stressstrain, stress-time, strain-time. Obviously, it will be extremely important to establish a process for maintaining consistency of coordinate systems between measured data and simulation data. This is particularly true when one considers composite materials and multiscale modeling. Consequently, a default coordinate system has been established within the NASA GRC GRANTA MI ${ }^{\bullet}$ database.

Lastly, there is 'References' section containing general notes, links to specific applications (which are stored in the Applications Table) and associated reports which are stored in the Reference Table within the NASA GRC GRANTA MI ${ }^{\circ}$ database. Note that the word 'links' appears in the column associated with type - to indicate that this 'attribute' is merely the name of the link within $\mathrm{MI}^{\circ}$ and not an actual attribute type itself.

Now given the ability to store simulation data (which can be significantly more voluminous than experimental) the next key question for an organization to address is how much of this type of information do they store. Do they mandate that all simulation data be captured or only those attached to a final product. Coming from a research organization, our current thought is to only capture those simulations associated with a final product - in our case, the product is published works. Such a decision is a difficult but extremely important and necessary one to make, as clearly a trade-off exists between the cost of data acquisition, storage, maintenance, and dissemination and the current and future value of the data being collected. This trade is extremely difficult to make $a$ priori as one oftentimes does not comprehend the importance of the data/information until after some time has elapsed and the window of opportunity has passed. Consequently, the desire is to collect as much information as possible at the time to avoid being in an 'if only I had ...' situation.

\section{Conclusions}

ICME is an integrated approach to the design of products, and the materials that comprise them, by linking various length scale-specific relationships across the scales, from processing all the way to product performance. A key ingredient is the linkage with manufacturing processes, which produce internal material structures, and in turn influence material properties and allowables, thus enabling tailoring (engineering) of both material and structure to specific industrial applications. As models become more accurate, their complexity tends to increase, and they rely less and less on simplifying assumptions. This complexity drives the need for more data to be measured, predicted, compared, stored, and tracked. Further, the goals of ICME, to link model results and experiments at multiple scales, drives the need for data/metadata and contextual linkage so that knowledge can be both captured and discovered. This underscores the value 
of a robust dynamic information management system enabling management of changing proprietary data alongside reference data collections, while ensuring consistency, quality, applicability, and full traceability. Often overlooked as a 'mere database,' this information management system should be viewed as a 'necessary' or an 'enabling' infrastructural aspect to ICME.

The benefits of a robust information management infrastructure are threefold. Firstly, it enables the capture, analysis, dissemination, and maintenance of various types of data (both experimental and virtual) for all materials with full traceability and security. Secondly, it will facilitate in the verification and validation of model output and certification of toolsets at multiple length scales, and thirdly, the establishment of input/output protocols enables the seamless integration of toolsets with optimization algorithms to provide final linkage of processing to performance criteria - thus making ICME a reality.

In this paper, we have taken the first step in articulating and implementing a robust ICME schema that incorporates 1) microstructure characteristic specifications, 2) material and model pedigree infrastructure for integrating experimental data with virtual data resulting from simulation models being applied at various levels of scales, and 3) attributes identifying the specific software tool(s) utilized. Further, some of the key requirements for best practice in materials informatics (both real and virtual data), were discussed that will enable organizations to effectively respond to the demands of new material and engineering applications and the pressures of operating in a globalized engineering environment. However, many hurdles (e.g., statistics, uncertainty, and optimization) are yet to be overcome, and further challenges (e.g., data quality evaluation and characterization, data error minimization and prevention, organizational and financial challenges) are to be expected, particularly in the area of ICME information management. However, these challenges are likely to be met as materials information management becomes mainstream and as more organizations demonstrate a return on their investment in technology in this area.

Competing interests

The authors declare that they have no competing interests.

Authors' contribution

SMA conceived of schema, associated attributes and drafted manuscript; FAH as database administrator implemented schema; BAB and EJP as MAC/GMC code developers supported the implementation of composite table attributes and associated composite examples. All authors read and approved the final manuscript.

\section{Acknowledgements}

The first two authors are grateful to their colleagues in the MDMC for many useful discussions on the key issues addressed in this paper.

Received: 6 November 2014 Accepted: 26 January 2015

Published online: 21 March 2015

\section{References}

1. Council NR (2008) Integrated Computational Materials Engineering: a transformational discipline for improved competitiveness and national security. National Academies Press, Washington, DC

2. Marsden W, Cebon D, Cope E (2011) Managing multi-scale material data for access within ICME environments. In: Arnold SM, Wong T (eds) Tools, models, databases, and simulation tools developed and needed to realize the vision of Integrated Computational Materials Engineering. ASM International, Materials Park, $\mathrm{OH}$

3. Arnold SM, Holland FA, Gabb T, Nathal M, Wong T (2013) The coming ICME data tsunami and what can be done. 54th AIAAJASME/ASCE/AHS/ACS structures, structural dynamics, materials conference, Boston, MA., 23-27 Apr 2013

4. Arnold SM (2006) Paradigm shift in data content and informatics infrastructure required for generalized constitutive modeling of materials behavior. MRS Bull 31(12):1013-1021

5. Cebon D, Ashby MF (2006) Engineering materials informatics. MRS Bull 31(12):1004-1012 
6. Arnold SM, Holland FA, Bednarcyk BA (2014) Robust informatics infrastructure required for ICME: combining virtual and experimental data. In: 55th AIAA/ASMe/ASCE/AHS/SC structures, structural dynamics, and materials conference. National Harbor, Maryland, pp 13-17, Jan 2014, AIAA-2014-04

7. Open MDAO. http://openmdao.org, 01/15/15.

8. Ren W, Cebon D, Arnold SM (2009) Effective materials property information management for the 21st century. In: Proceedings of PVP2009, 2009 ASME pressure vessels and piping division conference. Czech Republic, Prague, pp 26-30, July 2009, PVP2009-77314, pp. 1-10

9. Collier Research Corporation makers of HyperSizer. www.HyperSizer.com, Accessed 15 January 2015.

10. Simulia Abaqus, a Dassault Systemes subsidiary. http://www.3ds.com/products-services/simulia/products/abaqus/. Accessed 15 January 2015.

11. Aboudi J, Arnold SM, Bednarcyk BA (2013) Micromechanics of composite materials: a generalized multiscale analysis approach. Elsevier, Oxford, UK

12. Sullivan RW, Arnold SM (2011) An annotative review of multiscale modeling and its application to scales inherent in the field of ICME. In: Arnold SM, Wong TT (eds) Models, databases, and simulation tools needed for the realization of Integrated Computational Material Engineering. ASM International, Materials Park, OH, pp 6-23

13. Bednarcyk BA, Arnold SM. MAC/GMC 4.0 user's manual - keywords manual. NASA/TM-2002-212077/NOL2, 2002a, NASA Glenn Research Center, Cleveland, OH, USA.

14. Bednarcyk BA, Arnold SM. MAC/GMC 4.0 user's manual - example problems manual. NASA/TM-2002-212077/NOL3, 2002b, NASA Glenn Research Center, Cleveland, OH, USA.

15. Bednarcyk BA, Arnold SM (2006) A framework for performing multiscale stochastic progressive failure analysis of composite structures. In: Proceedings of the 2006 Abaqus user's conference, 23-25 May 2006. MA, Boston

16. Granta Design Limited. http://www.grantadesign.com/. Accessed 19 Mar 2013.

17. Official website of Materials Data Management Consortium. http://mdmc.net. Accessed 19 March 2013.

18. Dowling NE (1999) Mechanical behavior of materials: engineering methods for deformation, fracture, and fatigue. Prentice Hall, New Jersey

19. Lemaitre J, Chaboche JL (1990) Mechanics of solid materials. Cambridge University Press, Cambridge, UK

20. Skrzypek J, Hetnarski R (2000) Plasticity and creep, theory, examples, and problems. CRC Press, Boca Raton, FL, USA

21. Lemaitre J (2001) Handbook of materials behavior models. Academic Press, San Diego, USA

Submit your manuscript to a SpringerOpen ${ }^{\odot}$ journal and benefit from:

- Convenient online submission

- Rigorous peer review

- Immediate publication on acceptance

- Open access: articles freely available online

- High visibility within the field

- Retaining the copyright to your article

Submit your next manuscript at $>$ springeropen.com 